\title{
Overcoming Localization Errors due to Node Power Drooping in a Wireless Sensor Network
}

\author{
Safdar Abbas Khan, Boubaker Daachi, and Karim Djouani
}

\begin{abstract}
Received Signal Strength Indication (RSSI) plays a vital role in the range-free localization of sensor nodes in a wireless sensor network and a good amount of research has been made in this regard. One important factor is the battery voltage of the nodes (i.e., the MICAz sensors) which is not taken into account in the existing literature. As battery voltage level performs an indispensable role for the position estimation of sensor nodes through anchor nodes therefore, in this paper, we take into a account this crucial factor and propose an algorithm that overcomes the problem of decaying battery. We show the results, in terms of more precise localization of sensor nodes through simulation. This work is an extension to [1] and now we also use neural network to overcome the localization errors generated due to gradual battery voltage drooping.
\end{abstract}

Keywords-Wireless sensor networks, RSSI, localization, range-free scheme, energy considerations, neural networks.

\section{INTRODUCTION}

W IRELESS Sensor Networks (WSNs) have become important in the fields of military defence and environmental sciences. Their applications are also found in home and ubiquitous environments. A wireless sensor network is a network of distributed nodes that monitor the physical changes like temperature, pressure, vibrations, and motion/breaches at the desired locality, to name a few, [2]-[4]. The nodes are fully equipped to measure these changes. After an observation is made it travels in the form of data packets from node to node till it reaches its destination. For this purpose the sensors are also equipped with transceiver antennas. To perform these activities the nodes use energy provided by the attached battery.

One of the many important issues in the WSNs is the localization [5]-[7]. There are certain applications of the WSNs, e.g., environmental monitoring like forest fire observation, and like intrusion, in which the location of the information source is very important. The received data is meaningless unless the position of the event occurrence is known. There are two types of the localization schemes: range-based and range-free, [5]-[7]. In a WSN there are two types of the nodes: anchors and sensors. Anchors are equipped to know their position/location, either by GPS or by pre-configuration [8]-[10]. But for sensors, neither they are equipped with GPS nor their locations are pre-configured. In range-based schemes sensors are localized with the known positions of anchors by measuring the angle of arrival (AoA), time of arrival (ToA), or

S. A. Khan, B. Daachi, and K. Djouani are with the LiSSi Laboratory, EA-3956 of University of Paris-East Créteil, Val-de-Marne, France (e-mails: \{safdar-abbas.khan, daachi, djouani\}@u-pec.fr).

$\mathrm{K}$. Djouani is a full professor at UPEC and is also with the F'SATI Laboratory of Tshwane University of Technology, South Africa. time difference of arrival (TDoA) [11], [12]. For this scheme to be applicable we need to employ certain devices in order to measure one or all of the three quantities. In range-free schemes sensors are localized with the help of known positions of anchors but without the use of AoA, ToA, or TDoA. Most of the time the distance between a sensor and an anchor is calculated using RSSI.

Figure 1 shows a MICAz sensor that is used in our experiments. In [13] it is shown that with the passage of time these sensors start losing battery voltage. The drop in the battery

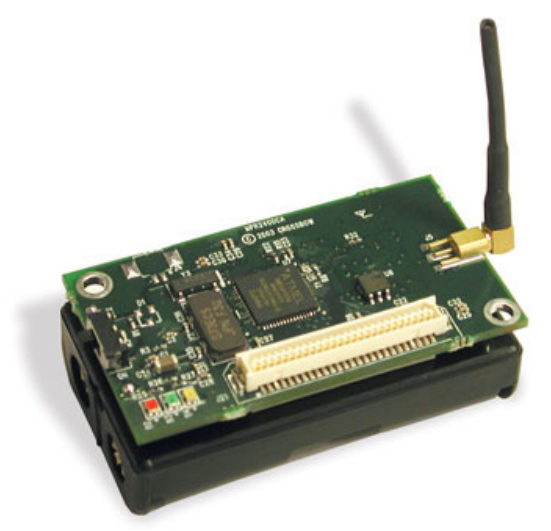

Fig. 1. MICAz mote for which the idea is proposed. These motes loose their battery voltage with the passage of time.

voltage of MICAz sensor is measured over a period of 200 hours and is graphically presented in fig. 2 . This drop in battery voltage may lead to erroneous sensor location.

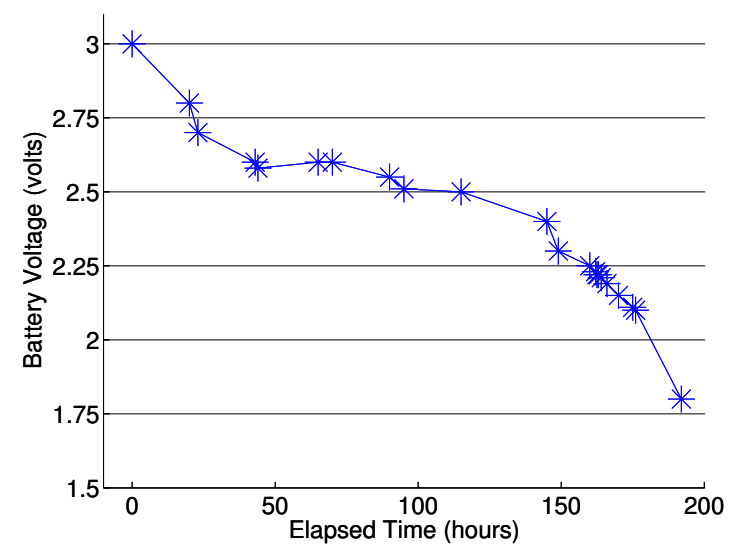

Fig. 2. Plot of the battery voltage of MICAz sensor against time over the period of two hundred hours. 
A range-free geometric approach towards the computation of the sensor nodes location is given in [8], [14]; where the estimated position of a sensor is the centroid of positions of connected anchors. If $\mathbf{r}_{1}, \mathbf{r}_{2}, \mathbf{r}_{3}, \cdots, \mathbf{r}_{n}$ are the positions of connected anchors to a particular sensor, then the estimated position of that sensor is given by (1).

$$
\mathbf{r}_{e s t}=\frac{1}{n} \sum_{i=1}^{n} \mathbf{r}_{i}
$$

In [9] an improved version of this geometric approach is given that utilizes the weighted average method. For a particular sensor, a weight, called edge weight, is assigned to a connected anchor according to its proximity. Suppose $w_{1}, w_{2}, w_{3}, \cdots, w_{n}$ are the weights assigned to connected anchors in reference to a sensor then the estimated position of the sensor is given by (2).

$$
\mathbf{r}_{e s t}=\frac{\sum_{i=1}^{n} w_{i} \mathbf{r}_{i}}{\sum_{i=1}^{n} w_{i}}
$$

There are two more techniques given in [5] for sensor localization. Fuzzy logic system (FLS) and genetic algorithm are used in one of them and the other is carried out by the implementation of neural networks. These techniques produce better results as compared to those presented in [8], [9].

In this article, the energy considerations [15] are also taken into account while calculating the edge weights of the anchors. The edge weights calculated from RSSI are less informative due to drooping battery voltage leading to misinterpretation about exact sensor location. So we need to introduce a compensation term that will recoup the edge weight. An algorithm is given to find that compensation term and to calculate the enhanced location of the sensors.

We also present a solution to this problem through the use of neural networks. Although a soft computing technique for localization is used by [5], [14] but they have not considered the energy decrease in node batteries. When a neural network is trained with real data, it can learn the pattern between the input variables and the output variables. In our case the input variables are the battery voltage, the time elapsed since the node is in working mode and the RSSI observed. The single output variable is the real distance. Note that the distance obtained by the RSSI distance model presented in section III could be erroneous. Therefore it is better to deal the relationship between the observed RSSI, voltage, time elapsed and the real distance through a neural network.

The rest of the article is arranged as follows: In section II we present our idea and we formulate the problem. In section III we present our solution and devise an algorithm for the better performance regarding the localization of the senors. We analyze and implement our solution by introducing the new parameter of battery voltage decay in section IV. The treatment of the problem by using neural network is given in section V. Finally section VI gives the conclusion, demonstrating that adhering systematic attention to the battery voltage decay results in elevated performance of sensor localization through anchor nodes in a WSN.

\section{Problem Formulation}

In WSN anchor nodes are fully aware of their position in the geographical region and we need to find the sensor node positions. Sensor positions are calculated based on the anchor positions. The transmission range of an anchor is assumed to be spherical which is reduced to a circular region in two dimensional space. By transmission range we mean an area where a node can detect another node. A sensor node assigns edge weights to anchor nodes by measuring strength in their respective ping signals. Thus we get an initial estimate of sensor position by (2). The greater the RSSI observed from an anchor, the greater the edge weight is assigned by the sensor. That is the edge weight assigned has a positive correlation with the RSSI. The battery voltage level is gradually drooped due to its usage by the signal emitter of the anchor node. This battery voltage decay results in the reduced power supply to the emitter. The emitter then sends signals with reduced strength and declined RSSI. This leads to a misinterpretation about the distance between the sensor and the anchor. The implication is obvious that the error in distance measure is increased with decaying battery. In this article we are concerned with the calculation of the edge weight which is invariant to the battery voltage level. Thus we need to find out the compensation term that is added to the measured edge weight so that the resulting edge weight is reported as constant by a sensor for a particular anchor irrespective of the battery voltage.

Suppose the relationship between the edge weight $w$ and the battery voltage $V$, for a particular anchor-sensor node pair is presented by (3).

$$
w=f(V)
$$

If $V_{0}$ is the maximum voltage, then the maximum edge weight is $w_{0}=f\left(V_{0}\right)$. As a matter of fact we should have $f(V)=w_{0}$ for all battery voltage levels $V$. Thus with the decay in battery voltage the difference, denoted by $\chi(V)=\left|f\left(V_{0}\right)-f(V)\right|$, between the real edge weight and the observed edge weight increases. Thus $\chi(V)$ is the compensation term that we shall add to the observed edge weight to get the real edge weight. We denote by $g(\cdot, \cdot)$ a function of two variables; the observed edge weight $w_{o b}$ and the battery voltage $V$ of the anchor node, returning the real edge weight.

$$
g\left(w_{o b}, V\right)=w_{o b}+\chi(V)
$$

First of all we try to find out the relationship between the time elapsed $t$ and the battery voltage $V$ of the MICAz mote. The degree two and degree three polynomial approximations of battery voltage as a function of time elapsed are respectively given by (5) and (6), and are graphically shown in fig. 3 . Table I shows the difference between polynomially approximated values and the observed battery voltage at various time instances measured in hours. The quadratic and cubic polynomial approximations are providing us with results close to the observed data. We shall use the degree two approximation as a trade-off between better performance and increasing computation complexity.

$$
\begin{aligned}
& V(t)=a_{22} t^{2}+a_{21} t+a_{20} \\
& V(t)=a_{33} t^{3}+a_{32} t^{2}+a_{31} t+a_{30}
\end{aligned}
$$


where

$$
\begin{aligned}
& a_{33}=-5.444 \times 10^{-7} \\
& a_{32}=1.426 \times 10^{-4} \quad a_{22}=-1.194 \times 10^{-5} \\
& a_{31}=-1.342 \times 10^{-2} \quad a_{21}=-1.914 \times 10^{-3} \\
& a_{30}=2.983 \quad a_{20}=2.817
\end{aligned}
$$

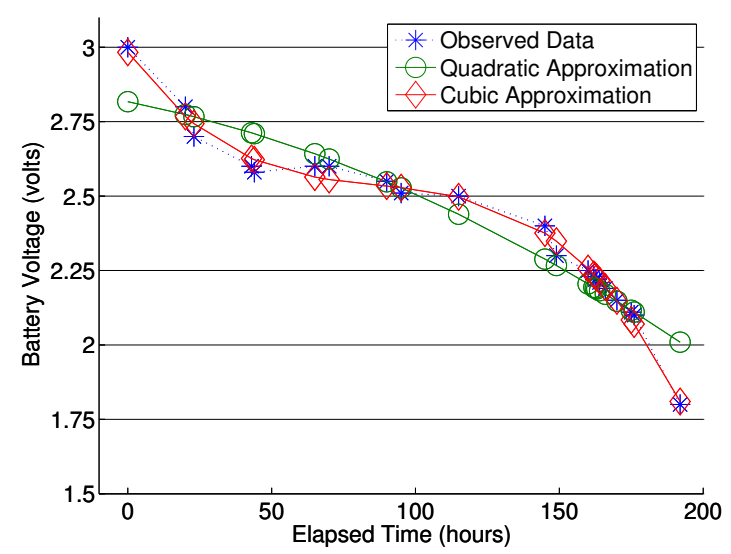

Fig. 3. The degree two and degree three polynomial approximations for the battery voltage against the time elapsed in hours.

An anchor node with low battery voltage will send signal with low RSSI. If this fact is avoided then sensor node shall conclude that the anchor node is at a farther distance than it really is. Now we shall compensate for this misinterpretation by the sensor node and we start our solution formulation in the next section.

TABLE I

DifFerence Between Polynomial APPRoXimated VAlues AND THE OBSERVED BATTERY VOLTAGE

\begin{tabular}{llll}
\hline Time & Linear Error & Quadratic Error & Cubic Error \\
\hline 20 & 0.08141 & 0.026056 & 0.0327152 \\
23 & 0.1801215 & 0.06666174 & 0.043151685 \\
43 & 0.2715315 & 0.11262094 & 0.026323789 \\
65 & 0.2620825 & 0.0421435 & 0.03632085 \\
90 & 0.301345 & 0.001974 & 0.0166076 \\
115 & 0.3406075 & 0.0610165 & 0.00237935 \\
149 & 0.5260045 & 0.03326594 & 0.048435164 \\
162 & 0.590421 & 0.03642136 & 0.006822557 \\
163 & 0.5999915 & 0.03221586 & 0.006620733 \\
166 & 0.628703 & 0.01974264 & 0.004518858 \\
170 & 0.666985 & 0.003446 & 0.0018972 \\
176 & 0.714408 & 0.01028256 & 0.029689254 \\
192 & 1.007536 & 0.20935584 & 0.009964173 \\
\hline
\end{tabular}

\section{Presentation of the Solution}

Consider one dimensional case with one anchor node at the origin and just one sensor node. By making sure that the battery of the anchor node is fully charged we calculate the edge weights by placing the sensor node at different positions as is shown in fig.4(a).

With the fully charged battery if the measured edge weights are $w_{1}, w_{2}, w_{3}, \cdots$, then we have the relationship $w_{1}>w_{2}>$ $w_{3}>\cdots$. At a later stage the weights measured by the sensor node are observed as shown in fig.4(b). Now the battery voltage level has drooped. Once again we have the

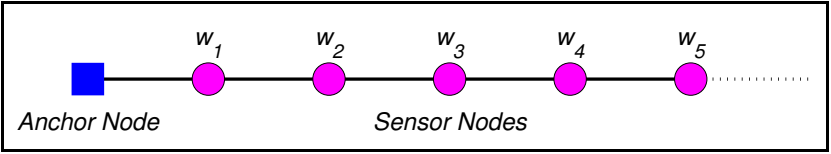

(a) Fully charged battery

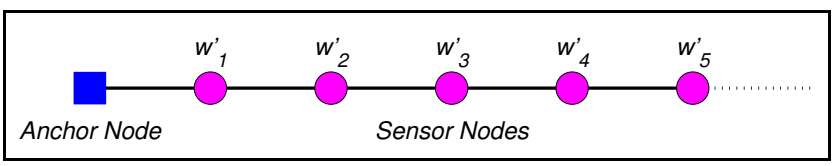

(b) Partially charged battery

Fig. 4. Weights measured by the sensor node at different positions at different voltage levels of the battery of the anchor node.

inequalities $w_{1}^{\prime}>w_{2}^{\prime}>w_{3}^{\prime}>\cdots$, but the signal strength is decreased. Thus we can have a situation in which $w_{i}^{\prime}<w_{i}$, (for $i=1,2,3,4$, or 5 ) whereby giving the misinterpretation about the location of the sensor as we know that the distance is constant. Although the location of the sensor is same but the edge weight is lower than its preceding case. The sensor shall conclude that it is farther from the anchor than it really is. This is because the RSSI is weak and low edge weight is calculated.

Now let us find the relationship between the battery voltage level and observed edge weight for a fixed distance between sensor-anchor pair. In an experiment the RSSI at different sensor-anchor distances was measured and the results are shown in fig. 5. The cubic polynomial approximation from

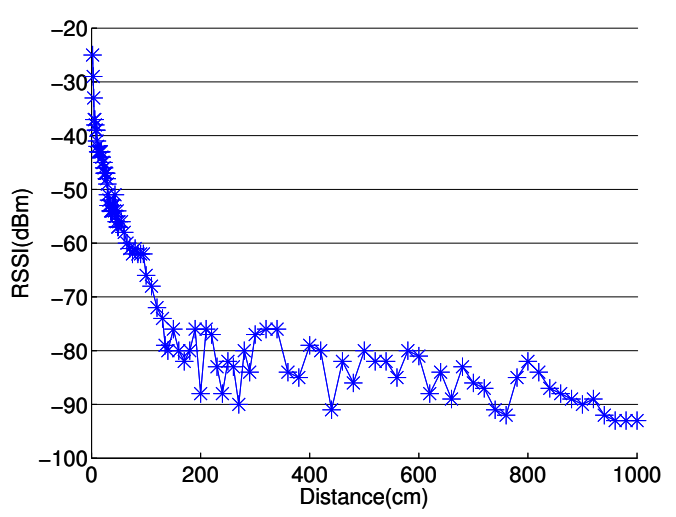

Fig. 5. RSSI measured against distance for MICAz sensor.

the observed data for distance $d$ in centimeters as a function of RSSI measured in $\mathrm{dBm}$ is given by (7).

$$
d=a_{3} \mathrm{RSSI}^{3}+a_{2} \mathrm{RSSI}^{2}+a_{1} \mathrm{RSSI}+a_{0}
$$

where

$$
\begin{array}{ll}
a_{3}=-0.007791 & a_{1}=-47.98 \\
a_{2}=-1.058 & a_{0}=-701.6
\end{array}
$$

The relation of RSSI in $\mathrm{dBm}$ to the power $P$ in $\mathrm{mW}$ is shown in $(8)$.

$$
\mathrm{RSSI}=10 \log _{10}(P)
$$

Edge weight is calculated from the RSSI which is the measure of the power of the signal. By the well known Watt's law the power, $P$, is the product of voltage, $V$, and current, $I$, i.e., 
TABLE II

Power In the MiCAz Mote Signal at Different Battery VOLTAGES

\begin{tabular}{cccc}
\hline $\begin{array}{c}\text { Voltage } \\
\text { (volts) }\end{array}$ & $\begin{array}{c}\text { Power } \\
\text { (Watts) }\end{array}$ & $\begin{array}{c}\text { Voltage } \\
\text { (volts) }\end{array}$ & $\begin{array}{c}\text { Power } \\
\text { (Watts) }\end{array}$ \\
\hline 1.54 & 2.37 & 2.51 & 6.3 \\
2.25 & 5.06 & 2.55 & 6.5 \\
1.85 & 3.42 & 2.59 & 6.71 \\
2.15 & 4.62 & 2.6 & 6.76 \\
2.05 & 4.2 & 2.6 & 6.76 \\
2.29 & 5.24 & 2.62 & 6.86 \\
2.43 & 5.9 & 2.66 & 7.08 \\
2.47 & 6.1 & 2.8 & 7.84 \\
2.49 & 6.2 & 2.94 & 8.64 \\
2.5 & 6.25 & 3 & 9 \\
\hline
\end{tabular}

$P=V I$ and by Ohm's law we have $V=I R$. Combining these two laws we get $P=\frac{V^{2}}{R}$. If the resistance remains constant, then we see that the power is directly proportional to the square of the voltage. By letting $R=1 \Omega$ we observe that with the decrease in the voltage of the battery of MICAz mote, there is decrease in the power of the sent signal as is shown in table II. Thus for a constant distance between the sensor-anchor pair the relationship between the power of the signal and the battery voltage is $P=V^{2}$. The observed edge weight decreases as the battery voltage droops. As a matter of fact we need the edge weight to remain constant for a fixed sensor-anchor pair distance.

As shown in fig. 5 , at the distance of $10 \mathrm{~cm}$, the RSSI of the signal is $-41 \mathrm{dBm}$ and the voltage is 3 volts. For this voltage the power in the signal using $P=10^{(R S S I / 10)}$ is $7.94 \times 10^{-5} \mathrm{~mW}$. For $V=3$ we have $P=7.94 \times 10^{-5}$ and we know that for $V=0, P=0$. As $P$ is proportional to the square of $V$, it means that 9 corresponds to $7.94 \times 10^{-5}$. Thus power of the sent signal as a function of battery voltage is given by (9) where $\alpha=8.82 \times 10^{-6}$. Figure 6 shows the plot of power in the signal in relation to the battery voltage.

$$
P(V)=\alpha V^{2}
$$

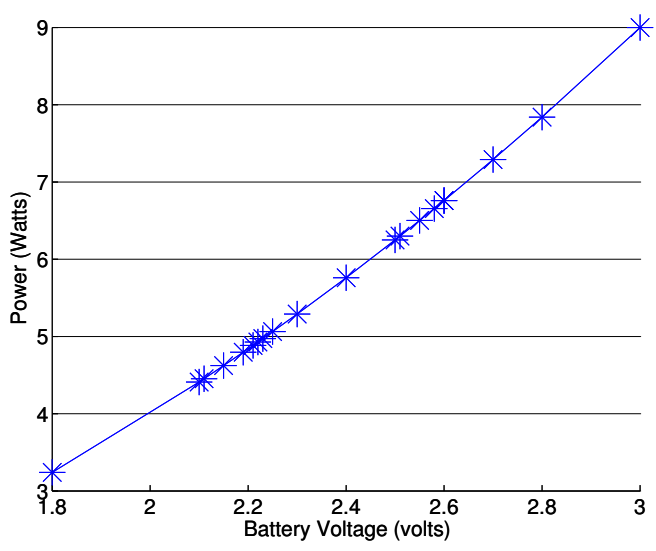

Fig. 6. The plot of power in signal as a function of battery voltage.

As edge weight $w$ is a function of RSSI, let us assume that it is an identity function:

$$
\begin{aligned}
& w=R S S I \\
& \text { (8) } \Longrightarrow w=10 \log _{10}(P) \\
& \text { (9) } \Longrightarrow w=10 \log _{10}\left(\alpha V^{2}\right) \\
& \therefore \quad \Longrightarrow w=f(V)=10 \log _{10}\left(\alpha V^{2}\right) \text {. }
\end{aligned}
$$

Hence the maximum edge weight is $w_{0}=-41$ and the compensation term is given by (10).

$$
\chi(V)=\left|w_{0}-10 \log _{10}\left(\alpha V^{2}\right)\right|
$$

The graph of the compensation term at different voltage levels is shown in fig. 7. The proposed technique is mentioned in algorithm 1 that computes the improved estimated position of the sensor nodes by compensating the edge weight loss due to battery voltage decay.

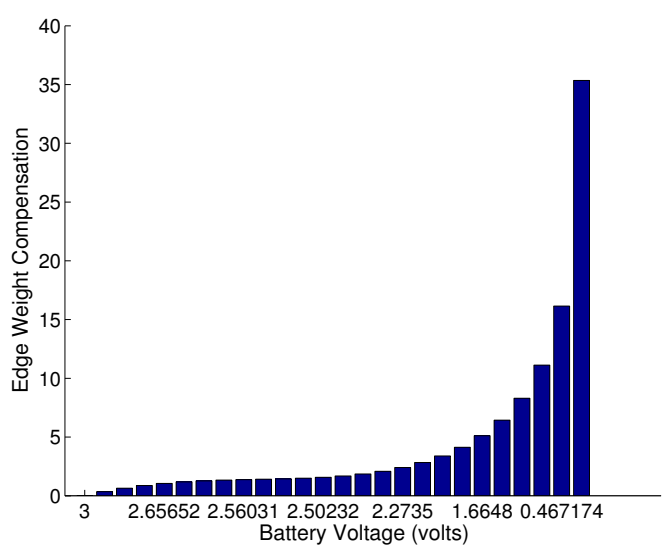

Fig. 7. Edge weight compensation at different battery voltages. It increases with the decrease in voltage.

$\overline{\text { Algorithm } 1 \text { Calculation of compensated edge weight and }}$ enhanced estimated position of sensor node

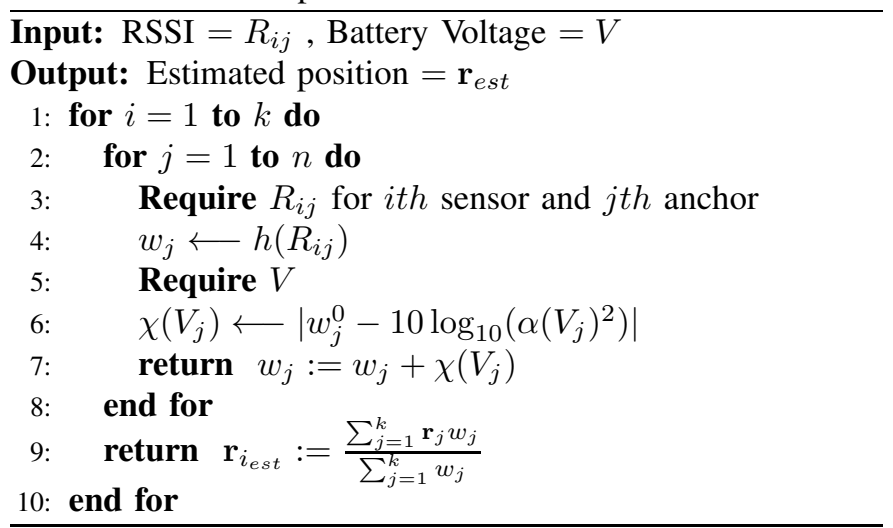

\section{Simulation Results}

Various simulations were performed using Matlab in order to validate the proposed algorithm 1. At any instant the compensation term for the observed edge weight is found as shown in (10). With the help of this compensation term the corrected edge weight is obtained as shown in line 7 of algorithm 1. Lastly with the help of the corrected edge weight the correct distance is calculated. In fig. 7 we have the graph of the edge weight compensation term plotted against decreasing battery voltage. We see that with the decrease in 
battery voltage the magnitude of the weight to be compensated is increased. If $w_{o b}$ represents the observed edge weight at any instant then the corrected edge weight $w$ is given by putting for corresponding values in (4) as shown in (11).

$$
w=g\left(w_{o b}, V\right)=w_{o b}+\left|w_{0}-10 \log _{10}\left(\alpha V^{2}\right)\right|
$$

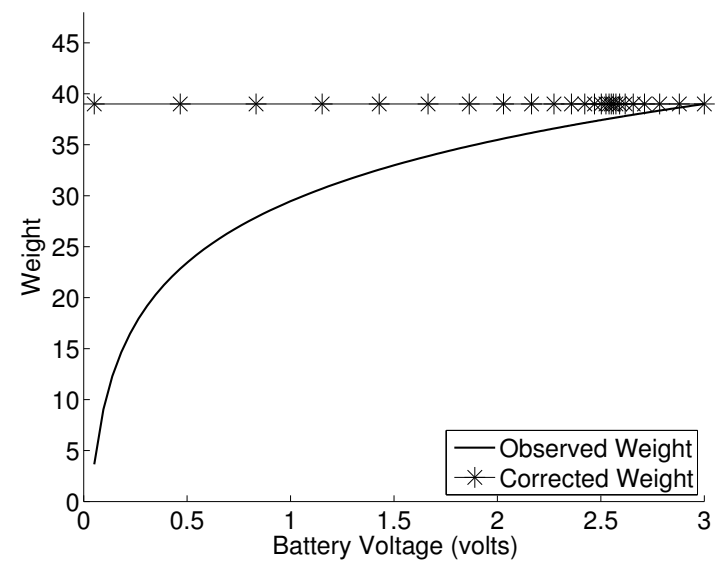

Fig. 8. Plot of observed and corrected edge weights against the battery voltage.

Figure 8 shows that there is remarkable decrease in the observed edge weight with the decrease in battery voltage. When the compensation term is found out and the corrected edge weight is computed we see that the corrected weight remains constant for a particular distance irrespective of the battery voltage. The difference between the observed distance and the corrected distance with the increase in time is shown in fig. 9. Finally the comparison of the three positions is shown in the fig. 10. Here we see that with the help of the compensation term added to the observed weight the localization of the concerned sensor node is improved.

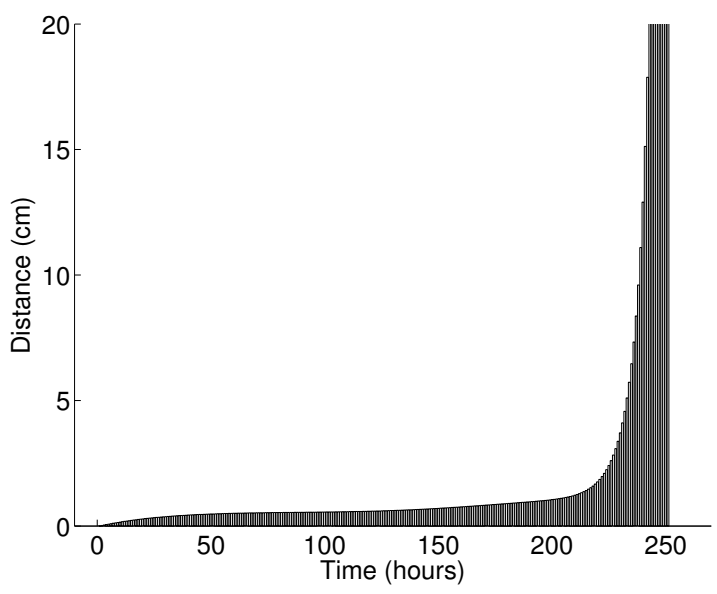

Fig. 9. Difference between the observed and corrected positions.

\section{Treatment through Neural Network}

Neural networks imitate human brain to perform intelligent tasks [16], [17]. A neural network is made to learn and

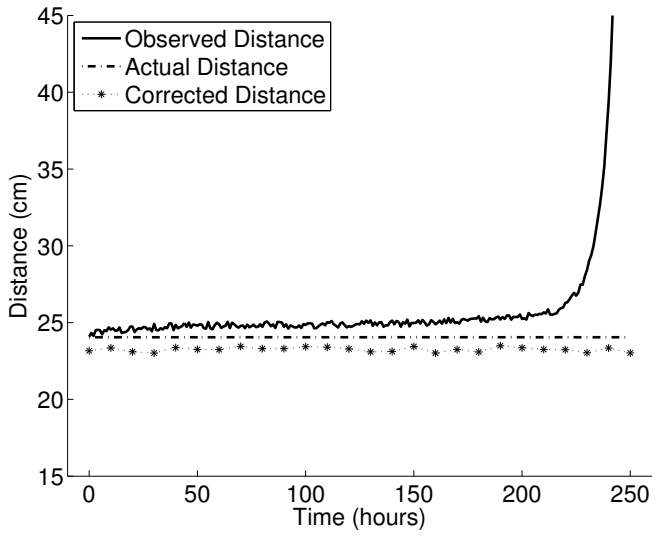

Fig. 10. The corrected distance improves the localization of the sensor nodes.

approximate the complicated relationships between input and output variables, and acquire knowledge about these relationships directly from the training data. A schematic diagram of the used neural network is shown in fig. 11. We have

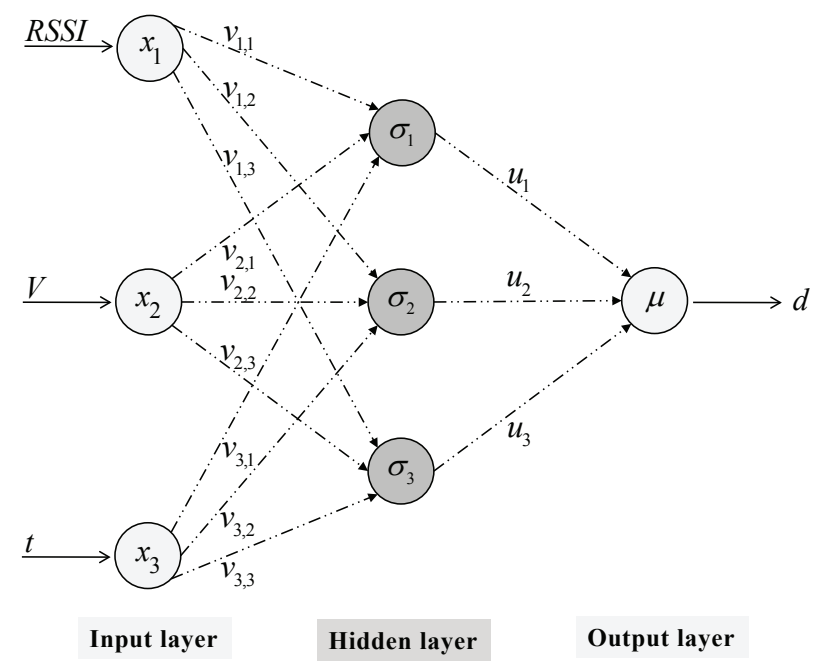

Fig. 11. Three layered neural network with three input variables and one output variable.

used a multilayer perceptron neural network with three layers: an input, a hidden and an output layer. The hidden layer has three neurons and the activation function of each of the neurons is the logsigmoid function. The components, in order, of the input vector $\mathbf{x}^{T}=\left(x_{1}, x_{2}, x_{3}\right)$ respectively are RSSI, voltage and the time elapsed. The square matrix of order three $\mathbf{V}=\left[v_{i, j}\right]$ represents the input-to-hidden layer weights. The activation function of each of the hidden layer neuron are denoted by $\sigma_{i}$ for $i=1,2$, or 3. Each of the $\sigma_{i}$ is the logsigmoid function. These three activation functions are represented by a vector $\boldsymbol{\sigma}^{T}=\left(\sigma_{1}, \sigma_{2}, \sigma_{3}\right)$. The vector $\mathbf{u}^{T}=\left(u_{1}, u_{2}, u_{3}\right)$ represent the hidden-to-output layer weight. The activation function of the output layer is denoted by $\mu$ and is the linear identity function. The single scalar 
output $d$ is the real distance between the nodes:

$$
d \approx \mu\left\{\sum_{j=1}^{3} u_{j} \sigma_{j}\left(\sum_{i=1}^{3} x_{i} v_{i, j}\right)\right\}
$$

In matrix form which is written as:

$$
F_{N N}(\mathbf{x})=\mu\left\{\mathbf{u}^{T} \boldsymbol{\sigma}\left(\mathbf{V}^{T} \mathbf{x}\right)\right\}
$$

One of the neural network simulation result is shown in fig. 12. Here we have obtained the estimated positions of the sensors with the help of distances obtained as an output of the neural network. These estimated positions are quite better than the positions obtained only from the observed RSSI. As

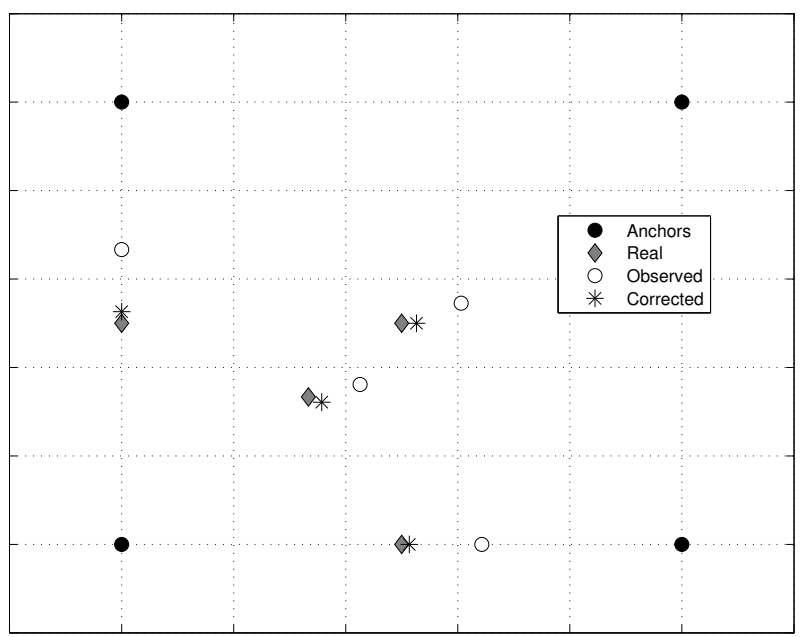

Fig. 12. Black dots are the anchors and the grey diamonds are the real positions, circles are the positions estimated from the observed RSSI and the black asterisks are the corrected positions.

shown in the figure there are four anchors at the vertices of a square of length $1.4 r$ where $r$ is the radius of transmission. Four senors are placed at different zones of connectivity. The network is already trained for the three variables. With the passage of time the error in the RSSI measure starts increasing. The observed RSSI is used to estimate the positions of the sensors. The average error in this case is 0.13 units. Now by using the distances obtained as the output variable of the neural network we get a better estimated position of the sensor nodes. The average error in the corrected estimated positions becomes 0.02 units. Hence the battery voltage consideration yields in a more reliable localization in wireless sensor networks.

\section{CONCLUSION}

In this article we have seen that the weight assigned to an anchor node due to observed RSSI when measured without paying attention to the battery level of that anchor node may lead to a misinterpretation about the distance between the respective anchor and sensor nodes. We have proposed a compensation term in the calculation of the edge weight that improves the accuracy of the distance between the concerned anchor and sensor nodes. With this added value of adherence to the battery voltage level of the anchor nodes, the localization of the sensor nodes in a WSN is improved. The battery voltage, the emitted power, and the received power are noticed. With the help of the proposed algorithm, before the estimation of the distance the compensation term, if needed, is added to the observed weight of the anchor node. Hence the uncertainty in the positions of the sensor nodes in a WSN, due to the proposed algorithm is reduced. The use of neural network techniques drastically reduces the computation complexity of the otherwise erroneous position estimation of the sensor nodes. The neural network also demonstrates that fact the battery voltage consideration gives an enhanced position estimation of the nodes as is shown in a simulation result. Thus the localization in wireless sensor network is improved when the time elapsed and the voltage droop are taken into account. The future works also include to tackle with the situation in which the loss in signal strength is not due to the battery voltage drooping.

\section{REFERENCES}

[1] S. A. Khan, B. Daachi, and K. Djouani, "Enhanced sensor localization through compensation of battery level decay," in Fifth International Conference on Broadband and Biomedical Communications 2010 (IB2Com), Malaga, Spain, December 2010, pp. 1-5.

[2] I. Akyildiz, W. Su, Y. Sankarasubramaniam, and E. Cayirci, "A survey on sensor networks," IEEE Communications Magazine, vol. 40, no. 8, pp. 102-114, 2002.

[3] I. F. Akyildiz, W. Su, Y. Sankarasubramaniam, and E. Cayirci, "Wireless sensor networks: a survey," Computer Networks, vol. 38, no. 4, pp. 393 422, 2002.

[4] P. Bergamo and G. Mazzini, "Localization in sensor networks with fading and mobility," in The Thirteenth IEEE International Symposium on Personal, Indoor and Mobile Radio Communications, 2002.

[5] S. Yun, J. Lee, W. Chung, E. Kim, and S. Kim, "A soft computing approach to localization in wireless sensor networks," Expert Systems with Applications, vol. 36, no. 4, pp. 7552-7561, 2009.

[6] S. Kim and O. Kwon, "Location estimation based on edge weights in wireless sensor networks," Journal of Korea Information and Communication Society, vol. 30, no. 10A, 2005.

[7] T. He, C. Huang, B. Blum, J. Stankovic, and T. Abdelzaher, "Range-free localization schemes for large scale sensor networks," in Proceedings of the Ninth Annual International MobiCom Conference. ACM, 2003.

[8] P. Bahl and V. Padmanabhan, "RADAR: An in-building RF-based user location and tracking system," in INFOCOM Nineteenth Annual Joint Conference of the IEEE Computer and Communications Societies, 2000, pp. 775-784.

[9] J. Hightower, R. Want, and G. Borriello, "SpotON: An indoor 3D location sensing technology based on RF signal strength," University of Washington, Computer Science and Engineering, Tech. Rep., 2000.

[10] T. Rappaport, J. Reed, and B. Woerner, "Position location using wireless communications on highways of the future," IEEE Communications Magazine, vol. 34, no. 10, pp. 33-41, 1996.

[11] D. Niculescu and B. Nath, "Ad hoc positioning system (APS) using AoA," in INFOCOM Twenty Second Annual Joint Conference of the IEEE Computer and Communications Societies, 2003.

[12] L. Cong and W. Zhuang, "Hybrid TDoA/AoA mobile user location for wideband CDMA cellular systems," IEEE Transactions on Wireless Communications, vol. 1, no. 3, 2002.

[13] C. T. Incorporation, "Battery life test for MICAz mote," Crossbow Technology Incorporation, Tech. Rep. [Online]. Available: http: //www.xbow.com/

[14] S. Yun, J. Lee, W. Chung, and E. Kim, "Centroid localization method in wireless sensor networks using TSK fuzzy modelling," in Eigth International Symposium on Advanced Intelligent Systems, Sokcho-City, Korea, 2007.

[15] G. Anastasi, M. Conti, M. D. Francesco, and A. Passarella, "Energy conservation in wireless sensor networks: a survey," Ad Hoc Networks, vol. 7 , no. 3, pp. 537-568, 2009

[16] M. T. Hagan, H. B. Demuth, and M. Beale, Neural Network Design PWS Publishing Company, 1996.

[17] C. M. Bishop, Neural Networks for Pattern Recognition. Oxford University Press Inc., New York., 1996. 